\title{
Automated Multidisciplinary Optimization of a Transonic Axial Compressor
}

\author{
U. Siller* \\ C. Voß $ß^{\dagger}$ \\ E. Nicke \\ German Aerospace Center (DLR), Institute of Propulsion Technology, Cologne, 51147, Germany
}

\begin{abstract}
The current paper describes DLR's optimizer AutoOpti, the implementation of the metamodel "Kriging" as accelerating technique, and the process chain in the automated, multidisciplinary optimization of fans and compressors on basis of a recent full stage optimization of a highly loaded, transonic axial compressor. Methods and strategies for an aerodynamic performance map optimization coupled with a finite element analysis on the structural side are presented. The high number of 231 free design parameters, a very limited number of CFD simulations, and conflicting demands both within the aerodynamic requirements and between the disciplines are a challenging optimization task. To navigate such a multi-dimensional search space, metamodels have successfully been used as accelerating technique. Using four aerodynamic operating points at two rotational speeds allows adjusting a required stability margin and optimizing the working line performance under this constraint. The investigated compressor concept is a highly loaded transonic stage with a single row rotor and a tandem stator, designed for a very high total pressure ratio.
\end{abstract}

\section{A. Introduction}

$\mathrm{C}$ ompressors for aircraft engines are constantly developed towards higher aerodynamic loading to reduce the installation length, weight, and number of parts with no degradation in efficiency. This leads to more complex geometries and consequently to more complex flow structures. An automated optimization approach is to be preferred in order to take advantage of new design freedoms, while reducing or at least maintaining development time. Automated optimization is also suggested by recent progress in simulation technologies in several fields such as steady and unsteady computational fluid dynamics (CFD), structural and thermal finite element analysis (FEM). Moreover, processors have become increasingly powerful, and parallel computing on huge clusters can be considered state of the art technology for CFD and FEM applications. Thus, it has become possible to employ optimization methods in the design of various parts of heavy duty gas turbines and aircraft engines, even when calculations require large computational resources.

\section{B. Optimizer AutoOpti}

Multiobjective Optimization Strategies in Turbomachinery Design

The simulation-speedup and the emergence of improved optimization algorithms nowadays enable the development and use of automatic optimization methodologies to perform complex multi-disciplinary and multiobjective optimization processes in turbomachinery design. Such automated, computer assisted design-concepts have the potential to:

- Create new design-ideas for turbomachinery components and support the engineer.

- Reduce the number of design iterations within and between different disciplines like aerodynamic, structural and thermal analysis.

- Generate design compromises between the disciplines.

- Improve gas turbine performance and stability.

- Reduce time and cost.

\footnotetext{
${ }^{*}$ Research Engineer, Fan and Compressor Department, Member AIAA

† Team Leader Optimization Group

${ }^{*}$ Head of the Fan and Compressor Department
} \\ American Institute of Aeronautics and Astronautics
}




\section{Evolutionary Algorithms, the Program AutoOpti}

Most of the newest multiobjective optimization methods are based on genetic or evolutionary algorithms (notation: EA) because of their potential to handle almost any kind of objectives (simple, very complex and even uncomputable objectives coming from non converged simulations).

Different schools of evolutionary algorithms have evolved during the last 40 years: genetic algorithms, mainly developed in the USA by J. H. Holland ${ }^{1}$ and evolutionary strategies, developed in Germany by I. Rechenberg ${ }^{2}$ and H.-P. Schwefel ${ }^{3}$. Each of these constitutes a different approach, but they are both inspired by the same principles of natural evolution. For a good introductory survey see D. B. Fogel ${ }^{4}$.

Evolutionary algorithms are stochastic search methods that mimic biological evolution. Most of them operate on a population of potential solutions applying the principle of survival of the fittest to produce better and better approximations to an unknown solution. At each generation, a new set of free parameters is created by the process of selecting individuals according to their level of fitness in the problem domain and breeding them together using operators borrowed from natural genetics. Just like in natural adaptation, this process leads to the evolution of individuals that are better suited to their environment than their predecessors.

\section{AutoOpti, the Basic Flowchart:}

The following flowchart in Figure 1 shows the basic structure of the MPI-parallelized multiobjective evolutionary algorithm AutoOpti which was developed at the Institute of Propulsion Technology in the past five years, with focus on turbomachinery applications.

The root-process (grey) contains the optimization process (right hand side of figure 1). In order to calculate the fitness of a member (i.e. a set of values of the free parameters), it hands the member over to a slave process (orange) and upon termination receives its fitness values in return. The new member is stored in the database, and the Pareto rank (for a definition see Ref. 5) is updated for all stored members. In the next step some members (notation: parents) are selected from the database based on their fitness values and Pareto rank for the production of a new

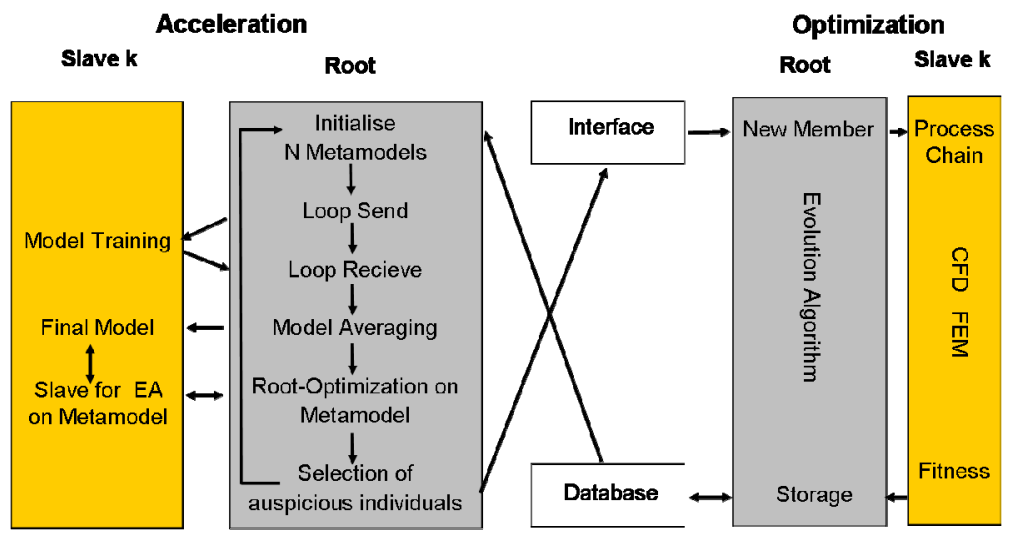
Figure 1. Optimization Metamodel Interaction in AutoOpti.

offspring. Several parents are recombined, using different operators like Mutation, Differential Evolution, and Crossover (see Ref. 4, 6,7), to produce the offspring. Now, the fitness values (and some other values of interest) of the offspring are computed by a slave-process, and the cycle is performed until the optimization criteria are reached or the user aborts the program.

The slave-process can be any transformation $\Phi$ of the following kind:

$\Phi: \quad \mathbb{R}^{N} \longrightarrow \mathbb{R}^{K}$, with $\mathrm{N}=\#\{$ Free Variables $\}$ and $\mathrm{K}=\#\{$ Objective Values $\}$

There are no other mathematical constraints to that operator $\Phi$ (for example to be continuous or differentiable). Thus, this method has a large field of application without any constraints on the specific process-chain.

In the next section, the most important special features of the program AutoOpti will be explained. These features distinguish AutoOpti from other optimization models and commercial tools. Most of them were developed in response to the enormous numerical effort of the process-chains in turbomachinery design (CFD and FEM for several operating points). Other features were implemented for a better handling and supervision of the optimization by the engineer.

Database, Restart, Asynchronous Communication, Constraint handling:

AutoOpti is not population based. The parents of a new offspring are selected from the current database of all evaluated members rather than from the previous population. The benefit of this method is an asynchronous communication between the root and the slave processes. In conventional evolutionary algorithms all slaves must wait until the slowest of them has finished before a new population can be generated. In contrast, the processor load in AutoOpti is almost $100 \%$ for all processes. 
Moreover, the database is essential for restart options and the setting up of metamodels (see next paragraph of this paper). For each member the set of free parameters, the objective values and all values of interest of a member (efficiency, mass flow, total pressure, total temperature, van-Mises stresses, ... ) are stored. This leads to a huge number of stored values (several hundred for typical turbomachinery applications). These values enable:

- A modification of the fitness functions at a restart of the optimization without any loss of information.

- Optimization observation by the engineer.

- Constraint handling: typical turbomachinery constraints can be monitored in consideration of these stored values. These constraints can be applied to the objectives and also to the values of interest. Unfulfilled constraints affect the Pareto rank and therefore the probability of selection of a member.

\section{Interface:}

The main drawback of EAs is the fact that they suffer from slow convergence because they use probabilistic recombination operators to control the step size and searching direction. It follows that - especially for expensive function evaluations - an EA typically requires a lot of CPU time.

To deal with this problem (see next chapter), and to make the optimization more controllable for the designer, an interface to the optimization process has been implemented (see figure1). If during the optimization the root process detects any external design input in the interface (either by a human engineer or an external algorithm), these designs will displace the evolutionarily created offspring. Thus, the optimization process can learn from external information and engineer know-how.

\section{Approximative Models}

To accelerate and improve the optimization process, different approximative models were used. Since approximations are models of a simulation which is itself a model of reality, they are often called metamodels. The terms approximation, surrogate model, response surface and metamodel will be used synonymously throughout this paper. The interaction between the original optimization and the approximative models is shown in Figure1.

While the original optimization is running (right hand side of figure 1), a second parallelized program is run for the training of metamodels and the optimization with these models (left hand side of figure 1) to find auspicious new members. Communication between these two programs occurs through the database (output of the original optimization and input for the metamodel training) and the interface (output of the metamodel optimization and input for the original optimization).

The basic idea of process acceleration using metamodels is quite easy to explain: The goal of using a surrogate model is to provide a functional relationship of acceptable fidelity to the "true" function with the added benefit of computational speed.

A metamodel is built using previously evaluated solutions in the search space and utilized to predict the fitness values of new candidate solutions. The transformation $\Phi$ (equation (1)) represents $\mathrm{K}$ unknown surfaces in an (N+1)dimensional space. Each evaluated member $\left(\mathrm{x}_{1}, \mathrm{x}_{2}, \ldots \ldots, \mathrm{x}_{\mathrm{N}}, \mathrm{f}_{1}, \mathrm{f}_{2}, \ldots \ldots \ldots, \mathrm{f}_{\mathrm{K}}\right)$ composed of the free parameters $\mathrm{x}_{\mathrm{i}}$ and the fitness values $\mathrm{f}_{\mathrm{j}}$, represents a point on these unknown surfaces. A metamodel is a second transformation

$\varphi: \quad \mathbb{R}^{N} \longrightarrow \mathbb{R}^{K}$ (K new surfaces in $\mathbb{R}^{N+1}$ ) which approximates or interpolates the previously evaluated members in the database in an appropriate manner. If the realization of the original $\Phi$ is very time consuming, like CFD or FEM processes, an optimization can perform much faster on its approximation $\varphi$.

The optimization on the metamodel in general strives for different goals than the original optimization. The infill sampling criterion, known as the expected improvement function, determines the optimization goal on the approximative model. It tends to choose the design points most likely to improve the accuracy of the model and/or have a better objective values than the current best points ${ }^{8}$ ).

\section{Curse of dimension}

The main challenge in metamodelling is given by the set up and the training of the models in high-dimensional search spaces (keyword: the curse of dimension). The curse of dimensionality is a term coined by Richard Bellman to describe the problem caused by the exponential increase in volume associated with adding extra dimensions to a (mathematical) space. For example, 100 evenly-spaced sample points suffice to sample a unit interval with no more than 0.01 distance between points; an equivalent sampling of a 10-dimensional unit hypercube with a lattice with a spacing of 0.01 between adjacent points would require $10^{20}$ sample points: thus, in some sense, the 10-dimensional hypercube can be said to be a factor of $10^{18}$ "larger" than the unit interval ${ }^{9}$ ). 
For typical turbomachinery optimization problems the number of available sample points for a metamodel (a few thousand evaluated members) is very small compared to the dimension of the search space (a few hundred free design parameters).

The details of how to build and exploit approximations effectively in high dimensional spaces, the selection of different infill sampling criteria like expected improvement, the improvement of the optimization schemes on the surrogate models (using gradient information), the averaging method of several models, etc., keep metamodel-based optimization a thriving research area.

The metamodels implemented in AutoOpti are Kriging models and Neural Networks. To clarify some typical difficulties, the Kriging procedure will be explained hereafter.

\section{Kriging:}

The Kriging model implemented in AutoOpti is outlined in Ref. 8. Let $\mathrm{M}$ be the number of sample points in the Database and $\mathrm{N}$ the number of free variables. A set of free parameters is denoted by $\vec{x}=\left(x_{1}, \ldots ., x_{N}\right)$, the vector of solutions (already evaluated members) for each objective is given by $\vec{y}_{s}=\left(y_{1}, \ldots, y_{M}\right)$. The correlation function between two sample points is called Cor, see eqn. (4), the mean value is $\hat{\beta}$ (3), the vector $\vec{r}$ (6) is the correlation vector between a new point and the samples $\vec{x}_{i}=\left(x_{i, 1}, \ldots ., x_{i, N}\right)$. The global model variance $\sigma^{2}$ is calculated by equation (7) and the mean error $s^{2}$ of the prediction $\hat{y}(\vec{x})$ (2) is given by equation (9). This error estimation $s^{2}$ is very important for the infill sampling criterion (expected improvement) in AutoOpti. The hyperparameters $\Theta_{k}$ and $p_{k}$ (4) and the regularization constant $\lambda$ (5) are all obtained by minimizing the right hand side of equation (8). This minimization yields the same results than maximizing the Likelihood, an optimization task itself with roughly twice the number of free parameters $(2 \mathrm{~N}+1)$ than the dimension of the original sample points. For simplification, all the $p_{k}$ are determined by the same value $p$. Thus, the number of hyperparameters to be determined is reduced to $\mathrm{N}+2$. The minimization procedure is called the training of the Kriging-model and uses the gradients of equation (8) with respect to the remaining model parameters $\Theta_{k}, p$ and $\lambda$. Difficulties during the training are the numerically expensive matrix inversion and the fact that minimizing (8) may lead to ill conditioned correlation matrices $R$.

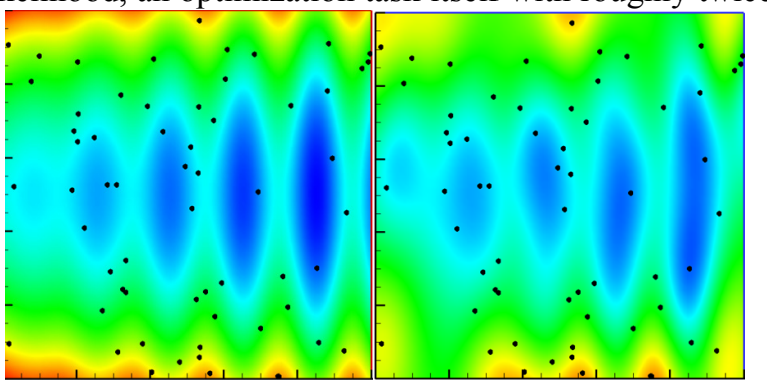

Figure 2. Kriging approximation in $\circ^{3}$

$$
\begin{aligned}
& \hat{y}(\vec{x})=\hat{\beta}+\vec{r}^{T}(\vec{x}) \cdot \vec{R}^{-1}(\vec{\Theta}, \vec{p}) \cdot\left(\vec{y}_{s}-\hat{\beta} \cdot \overrightarrow{1}\right) \quad, \vec{y}_{s}=\left(y_{1}, \ldots, y_{M}\right) \\
& \hat{\beta}=\frac{\overrightarrow{1} \cdot \vec{R}^{-1}(\vec{\Theta}, \vec{p}) \cdot \vec{y}_{s}}{\overrightarrow{1} \cdot \vec{R}^{-1}(\vec{\Theta}, \vec{p}) \cdot \overrightarrow{1}} \\
& \operatorname{Cor}\left(\vec{x}_{i}, \vec{x}_{j}\right)=\exp \left(-\sum_{k=1}^{N} \Theta_{k}\left|x_{i, k}-x_{j, k}\right|^{p_{k}}\right) \\
& R_{i, j}(\vec{\Theta}, \vec{p}, \lambda)=\operatorname{Cor}\left(\vec{x}_{i}, \vec{x}_{j}\right)+\lambda \cdot \delta_{i, j} \\
& \vec{r}^{T}(\vec{x})=\left(\operatorname{Cor}\left(\vec{x}, \vec{x}_{1}\right), \ldots \ldots, \operatorname{Cor}\left(\vec{x}, \vec{x}_{N}\right)\right)^{T} \\
& \sigma^{2}=M \cdot\left(\vec{y}_{s}-\hat{\beta} \cdot \overrightarrow{1}\right) \cdot \vec{R}^{-1}(\vec{\Theta}, \vec{p}) \cdot\left(\vec{y}_{s}-\hat{\beta} \cdot \overrightarrow{1}\right) \\
& \operatorname{MinLik}^{2}=\sigma^{2} \cdot \operatorname{det}\left(R_{i, j}(\vec{\Theta}, \vec{p}, \lambda)\right)^{1 / m} \\
& s^{2}(\vec{x})=\sigma^{2} \cdot\left[1+\lambda-\vec{r}^{T} \cdot \vec{R}^{-1}(\vec{\Theta}, \vec{p}) \cdot \vec{r}+\frac{\left(1-\overrightarrow{1} \cdot \vec{R}^{-1}(\vec{\Theta}, \vec{p}) \cdot \overrightarrow{1}\right)^{2}}{\overrightarrow{1} \cdot \vec{R}^{-1}(\vec{\Theta}, \vec{p}) \cdot \overrightarrow{1}}\right]
\end{aligned}
$$

Figure 2 shows on the left the contour plot of a simple function $f: \mathbb{R}^{2} \rightarrow \mathbb{R}$. The black points are the sample points, selected randomly on the surface $(x, y, f(x, y))$. The contour plot of the Kriging approximation, trained to approximate these sample points, is shown on the right hand side of figure 2 . 


\section{Automated and Multidisciplinary Optimization of a Transonic Axial Compressor}

The overall task of this optimization is a compressor stage geometry which promises the best possible aerodynamic compromise, based on multiple operating points to account for all essential performance map attributes. Structural and fabricational features are also to be considered. Key design parameters of this compressor stage design are listed in Table 1, figure 3 gives an idea of the blading geometries. These requirements are to be fulfilled with a transonic compressor stage consisting of a rotor and a tandem stator. The aimed total pressure ratio is relatively high for the tip speed used, resulting in bladings with a low aspect and pitch-to-chord ratio and a very high aerodynamic loading.

The initial member for this optimization (performance map in figure 8) has been initially designed using in-house S2- and S1-procedures, manual design iterations and a few optimizations similar to the presented type. In the process, optimization strategies and methods were developed and substantial progress was made in the stage design. Several different stator configurations were investigated and the latest optimized configuration was altered in the stator region with very different blade numbers and the exit area was increased to generate an initial member with higher potential for use in the present optimization. With these modifications the good rotor performance of former configurations was maintained. However, flow separations in the supersonic hub region of the stator occurred together with higher than tolerated deviations of the working line mass flow rates and the exit swirl. The task of the automated optimization process was to solve these issues and to maximize overall performance.

In the following this process is described together with information about geometry handling and the numerical setup. An overview of this process is shown in figure 4 .

\begin{tabular}{|c|c|c|}
\hline \multicolumn{3}{|l|}{ Rotor } \\
\hline Rotor Number of Blades & \multicolumn{2}{|c|}{19} \\
\hline Relative Inlet Mach Number at Rotor Tip & \multicolumn{2}{|c|}{1.6} \\
\hline $2 \cdot c p \cdot\left(T_{t o t, e x i t}-T_{t o t, e n t r y}\right)$ & \multirow{2}{*}{\multicolumn{2}{|c|}{1.02}} \\
\hline (2) & & \\
\hline Specific Flow at Rotor Leading Edge $\left[\mathrm{kgs}^{-1} \mathrm{~m}^{-2}\right]$ & \multicolumn{2}{|c|}{190.5} \\
\hline Inlet Radius Ratio $\mathrm{r}_{\mathrm{Hub}} / \mathrm{r}_{\mathrm{Tip}}$ & \multicolumn{2}{|c|}{0.32} \\
\hline Rotor Mean Aspect Ratio & \multirow{2}{*}{\multicolumn{2}{|c|}{0.85}} \\
\hline Rotor Average Pitch/Chord & & 0.45 \\
\hline Stator & $\mathrm{I}$ & II \\
\hline StatorI/II Number of Blades & 57 & 57 \\
\hline Absolute Inlet Mach Number at StatorI Hub & \multicolumn{2}{|c|}{1.2} \\
\hline StatorI/II Mean Aspect Ratio & 2.2 & 1.8 \\
\hline StatorI/II Average Pitch/Chord & 0.65 & 0.7 \\
\hline
\end{tabular}

Table 1. Stage Key Design Parameters.

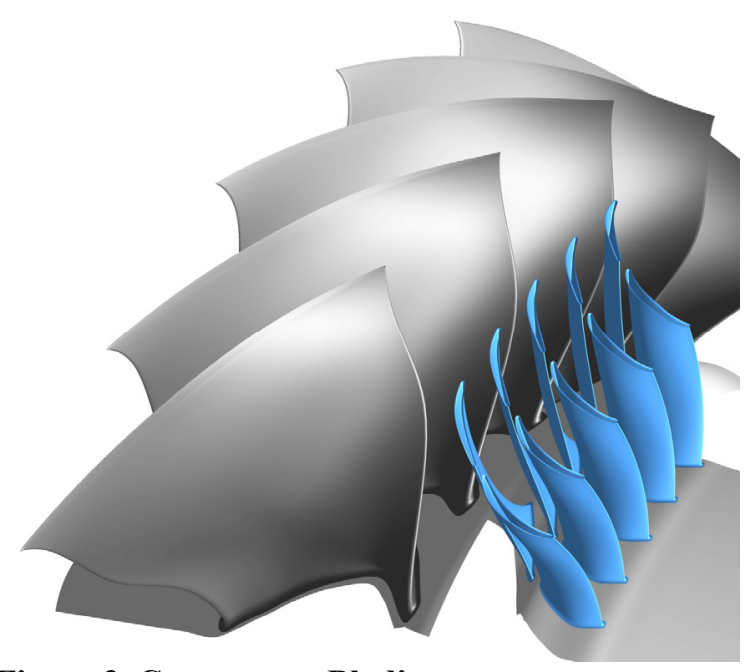

Figure 3. Compressor Bladings

\section{Range-scaling:}

Initially, all normalized variables from the optimization process of each slave have to be transformed to real scale values in order to fit the input requirement of the Bladegenerator.

\section{Blade and Duct Parameterization}

The duct geometry is parameterized by a series of interpolating spline control points for the hub and tip contour. As seen in Figure 5, there are free, fix and group points. The free points are allowed to shift radially within certain limits. A "group" is used for the exit duct, where all points downstream of the "group leader point" (casing), a free point, replicate the same shift while the corresponding points on the hub contour are shifted so as to keep the area of the exit

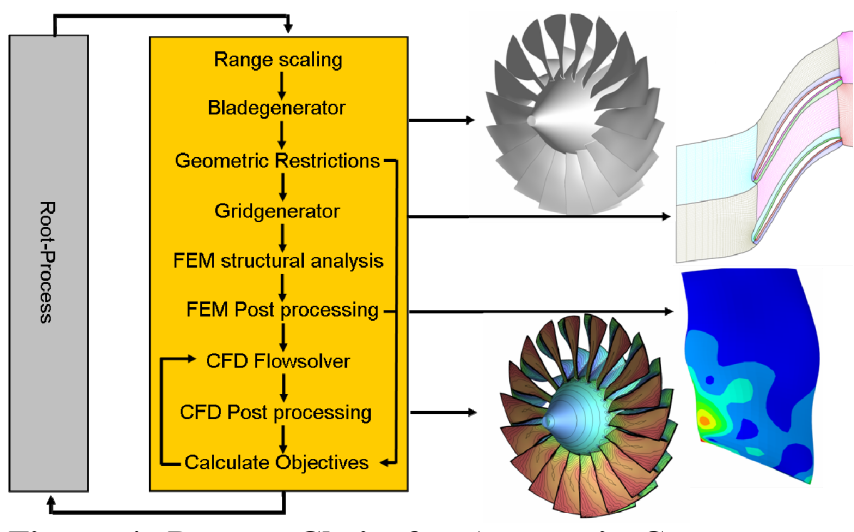

Figure 4. Process Chain for Automatic Compressor Optimization. duct constant. 
The presented optimization uses axis-symmetric hub and casing contours, but the process chain also supports highly complex, axis-asymmetric surfaces ${ }^{10}$.

The blade geometries are modeled and generated with the in-house program Bladegenerator based on parametrical B-spline curves and B-spline tensor product surfaces. Inputs are a coarse 2D-grid of "construction streamlines" and a set of profile parameters for every construction profile, which describe the 2D-shape of a profile in a streamline-based coordinate system (m', $\theta)$.

Profile parameters are:

- LE/TE angles,

- Stagger angle,

- LE/TE radius,

- Spline control points for the shape of the suction side,

- Thickness distribution to generate the profile pressure side on basis of the suction side. Inputs are the maximum profile thickness and its axial position (in the profile coordinate system), a fill factor for the front part of the profile (LE to maximum thickness) and a total profile fill factor. Additional parameters control the area distribution in the front and rear part of the profile and thereby a local curvature adjustment.

- Parameters that control the asymmetry

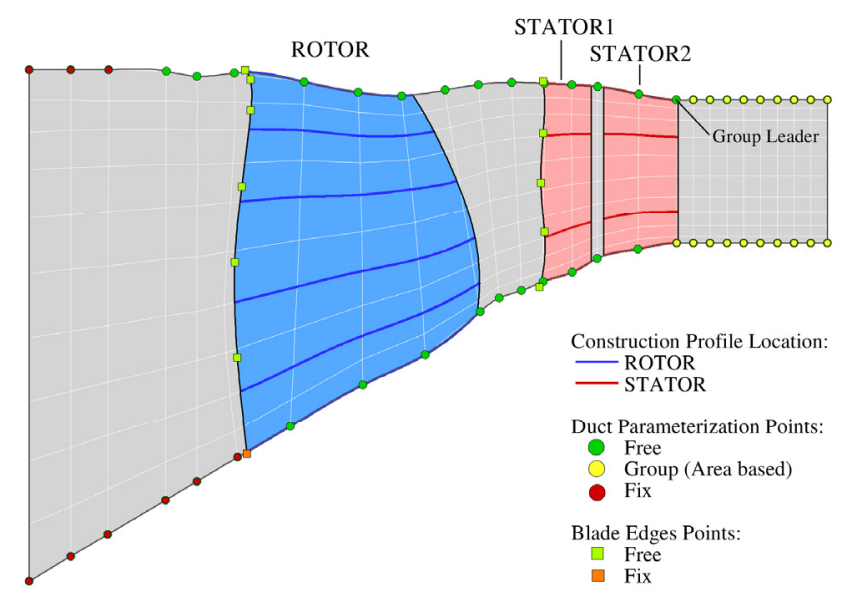

Figure 5. Parameterization of Duct, Blade Edges and Location of Construction Profiles. and shape of the edges.

Using a thickness distribution in an optimization is beneficial, since the profile area as an important mechanical parameter can be controlled by the maximum profile thickness and the profile fill factor.

Radial distributions can be used for all parameters. Using them is beneficial to reduce the number of free parameters, if the number and position of construction profiles results in a distribution of higher complexity than needed or intentionally to be permitted. Here a radial distribution was used for the rotor stagger angle (to avoid high local geometric gradients with negative impact on the manufacturability, especially close to the tip) and for parameters of the thickness distribution and LE asymmetry.

Once the profiles are generated in 2D, they are transformed to their construction streamlines in 3D together with a stacking law. The circumferential position of the rotor profiles on their rotational construction stream surfaces is defined by an adapted center of gravity stacking law. In axial direction the profiles of all blade rows are scaled to fit inside an axial domain, specified by a leading and trailing edge curve (see figure 5). The leading edge curve of rotor and statorI is subject of optimization with 6 free points for the rotor and 5 for statorI. The stator stacking law is trailing edge for statorI and leading edge for statorII.

The stator profiles are free to shift in circumferential direction except the hub profile of statorI. Thus the aerodynamically highly important relative position between statorI and statorII is considered.

For optimizing the mechanical balancing of the rotor blade in circumferential direction a linear radial distribution is used for the construction profile $\theta$-shift.

\section{Geometric Restrictions}

Properties of construction profiles, such as fill factor, maximum thickness and position, maximum chord length, and many more, can be subjected to geometric restrictions.

Having passed these restrictions, the 3D blade is generated and another set of radial geometric restrictions can be imposed, e.g. extremum and monotonicity checks for profile parameters and/or

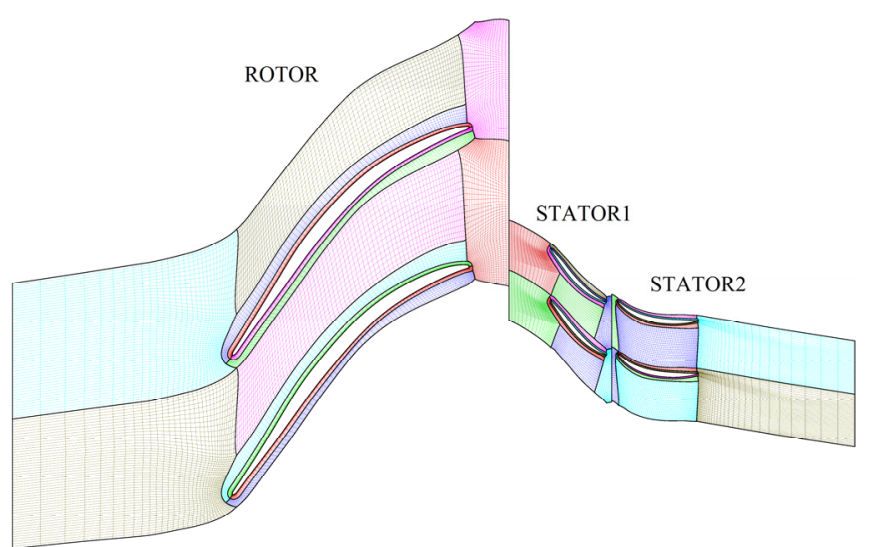

Figure 6. Topology of the Computational Grid. Iso-k-surface at midspan. 
calculated attributes. The optimization presented in this paper had no geometric restrictions; all demands were satisfied with a rotor FE analysis and the manner and combinations of setting free parameter limits.

\section{Grid Generation}

The automated grid generation in the optimization process is performed by an extension of CFD Norway's G3DMESH. The grid consists of 23 structured blocks with overall 1.3 million grid points in an O-C-3H topology for every blade row (Figure 6). The radial grid resolution consists of 64 grid layers with 11 layers in the rotor tip gap. The first grid distances at solid type boundaries are chosen for the use of a wall function (y+ 50).

Input for the grid generation process is a S2M-grid, generated by the optimizer based on the hub and casing contour, a given point distribution in an initial S2M-grid, and the blade surface geometry from the blade generation process. During the grid generation process fillet radii on rotor hub and stator hub/tip are also generated.

The blade rows are connected by unstructured interfaces, a mixing plane between rotor exit and statorI entry and an unstructured axial interface between the two stators.

\section{Flow Simulation}

All calculations reported are conducted with TRACE in the version 6.1.28, a cell-centered finite volume Reynoldsaveraged Navier-Stokes solver, which is being developed by the numerics group at the DLR Institute of Propulsion Technology in cooperation with MTU Aero Engines specifically for the simulation of turbomachinery flows. For the turbulence closure the two equation Wilcox k- $\omega$ model was used. Theory and methods of TRACE as well as code validation on the basis of experimental results can be found in Ref. 11, 12, and 13.

Beside TRACE two other flow solvers are supported: MISES from Drela and Youngren ${ }^{14}$ for profile section optimization and the inviscid through-flow method MAGELAN in a coupled simulation with TRACE by matching the flow information at interfaces between both CFD codes in an iterative procedure. For an optimization example using TRACE-MAGELAN see Ref. 15.

In turbomachinery CFD the desired operating point is typically set by a static pressure boundary condition at the compressor outlet panel. In an optimization with a limited number of operating points this approach has the penalty of generating neither comparable flow kinematics between different members nor information about the location of
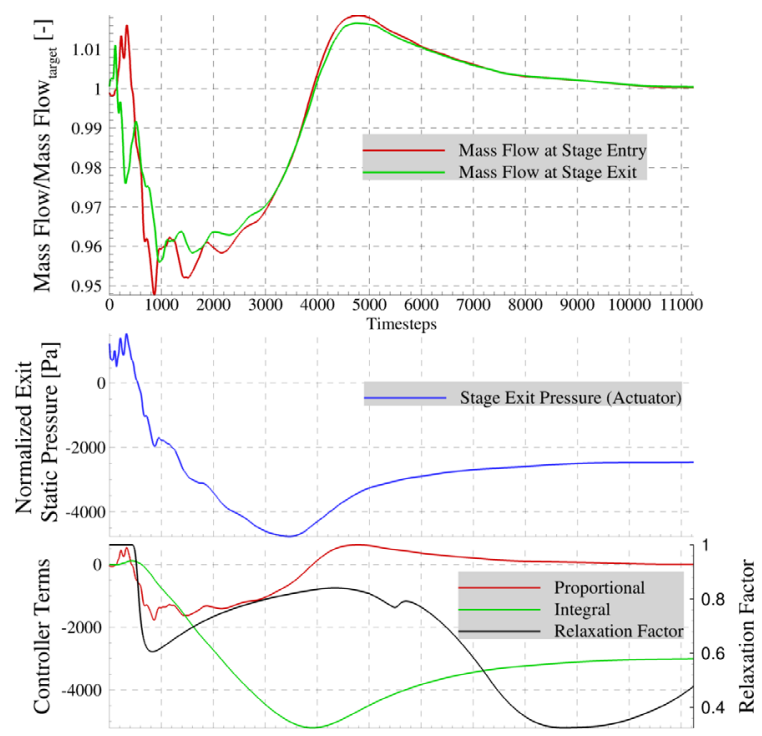

Figure 7. Mass Residuals, Stage Exit Static Pressure, and Controller Terms.

Mass controlled operating point at $100 \% \mathrm{rpm}$ close to the numerical stability limit.

the numerical stability limit.

This problem was solved by implementing a controller in the flow solver TRACE, which adjusts the exit static pressure for a desired mass flow rate. The algorithm is based on the widely used PID approach, extended by an adaptive sensitivity adjustment. This ensures faster convergence and adapts the controller settings in the case of mass flow oscillations. Figure 7 shows normalized mass flow residuals for the stage entry and exit panel, the pressure signal, set by the controller at the stage exit and the composition of this signal by the proportional and integral part together with the weighting factor, which scales the controller law by the sensitivity of the actual compressor and operating point.

\section{Finite Element Structural Analysis}

Two FEM Solvers are linked to the process chain. The commercial code PERMAS (INTES GmbH) and the open source code CalculiX. Static structural calculations and dynamic analysis for a Campbell diagram analysis are implemented in the process

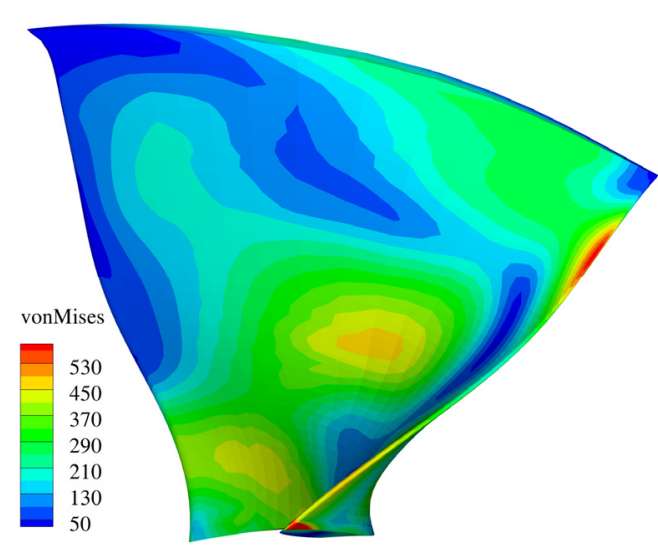

Figure 8. Finite Element Analysis. Von-Mises stress distribution in the Initial Member rotor blade (CalculiX). 
chain. After post processing some characteristic stress statistics are stored in the database.

These results can be used either as geometric constraint to discard geometries ahead of the much more expensive CFD simulations, or as input for an objective. In the presented optimization the maximum von-Mises stress is used as constraint. The stress distribution of the initial rotor blade is shown in figure 8. During optimization, the maximum tolerated stress limit is updated to stress values found for aerodynamically dominant members.

\section{Operating Points and Optimization Strategy}

Finding "the optimum" within the search space determined by the presented 231 free design parameters is impossible - especially when limited to just a few thousand fitness evaluations due to the expensive flow simulations in multiple CFD operating points. The complexity of the search space is somewhat reduced, because flow physics in turbomachines, e.g. on stream layers, leads to an interactional grouping of some parameters. A local objective "efficiency" on a given relative duct height is for instance dominated by the blading parameters, placed on the same blade height and of course the duct parameters.

Under these circumstances it is crucial to carefully select a set of free parameters which potentially solves the optimization problem. Furthermore the acceleration technique with response surfaces or metamodels has been

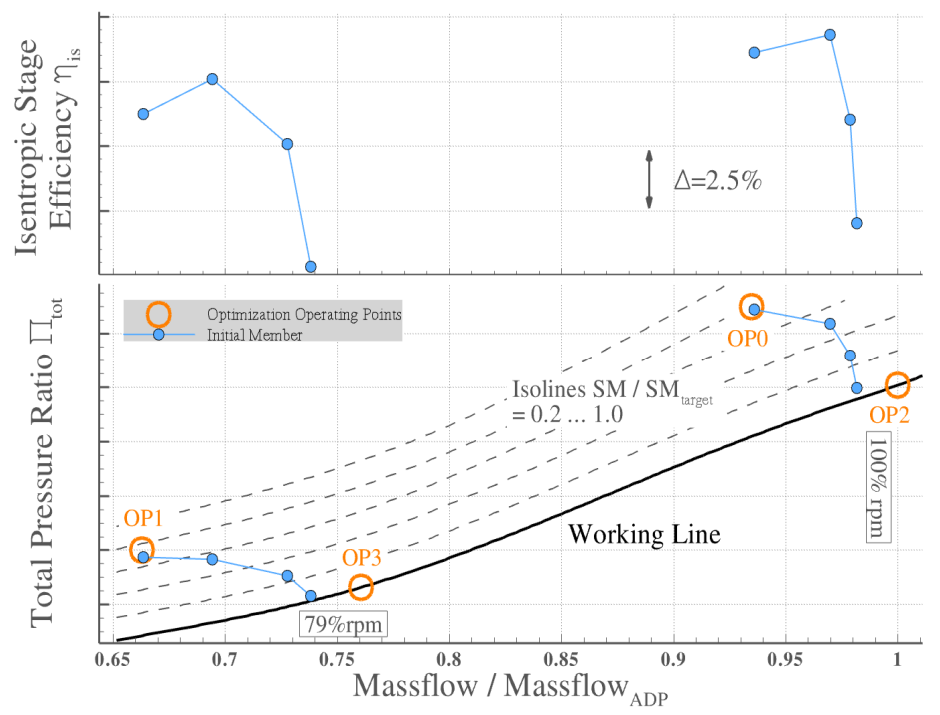

Figure 8. Aerodynamic Operating Points and Initial Member Performance Map extensively used to drive the optimization in small steps in the right direction.

In our process (figure 1) the optimization is fully controlled by the acceleration branch, since all members were created on the basis of an optimization on metamodels. Furthermore, a separate metamodel collective is trained for all flow-, performance- or even binary parameters like CFD convergence, needed for objective or constraint formulation. On these metamodels a multi objective optimization is conducted and a set of auspicious members selected. The frequency of this process is set by the time period needed to get a member trough all CFD and FE calculations and to make new information available in the database for the metamodel generation.

The presented optimization tries to include all essential performance map quantities, namely the stall margin and the working line performance by efficiency, total pressure ratio, mass flow rate, and stage exit swirl. Especially stall margin and efficiency are highly negatively correlated, which means that a higher stall margin decreases the maximum possible working line efficiency. Thus, at least two operating points at design rotational speed are required, one at the working line and the other close to stall. Aircraft engine compressors with a broad working range of rotational speeds need these operating conditions at least at one additional rotational speed to ensure sufficient off-design performance. Consequently, our optimization is conducted with four operating points (orange circles in figure 8). The operating points are calculated subsequently with decreasing criticality with respect to convergence:

- OP0: Near Stall at $100 \% \mathrm{rpm}$. Mass flow controlled on a rate, which is $6.5 \%$ lower than the targeted mass flow rate on the working line for the same rotational speed. This OP is near stall, thus a minimum stall margin can be derived by this point, the remaining reserve can not be determined.

- OP1: Near Stall at 79\% rpm. Mass flow controlled on a rate, which is $13.1 \%$ lower than the targeted mass flow rate on the working line for the same rotational speed. This point ensures the stall margin demands at part rotational speeds.

- OP2: Working line at $100 \% \mathrm{rpm}$ (ADP). A static pressure exit boundary condition is used to set the desired pressure ratio. Other important quantities are mass flow rate, efficiency, exit Mach number and stage exit swirl angle.

- OP3: Working line at $79 \% \mathrm{rpm}$. A static pressure exit boundary condition is used to set the desired pressure ratio. Other important quantities are mass flow rate and efficiency. 


\section{Objectives}

Two simple fitness functions are used: The average working line efficiency (derived from OP2 and OP3) and the average stall margin (derived from OP0 and OP1). The arithmetic mean of both quantities is used due to a very similar stall margin-efficiency correlation at design and part speed and the demand for a constant performance improvement in the working range.

$$
\begin{aligned}
& F 1=0.5 \cdot\left(-\eta_{i s, O P 2, \text { corrected }}-\eta_{i s, O P 3, \text { corrected }}\right) \quad \text { with } \quad \eta_{\text {is, OP } 2 / \mathrm{OP} 3, \text { corrected }}=\frac{\prod_{\text {total }, \text { OP } 2 / O P 3, \text { target }}^{\gamma}-1}{\frac{T_{\text {total }, \text { exit }}}{T_{\text {total }, \text { entry }}}-1} \\
& F 2=0.5 \cdot\left(-S M_{100 \% \text { rpm }}-S M_{79 \% \text { rpm }}\right) \quad \text { with } \quad S M_{100 \% \text { rpm } / 79 \% \text { rpm }}=\frac{\prod_{\text {total }, \text { OP } 0 / \text { OP } 1}-\prod_{\text {total,WorkingLine }}}{\prod_{\text {total,WorkingLine }}}
\end{aligned}
$$

The stage efficiency $\eta_{i s, O P 2 / O P 3, \text { corrected }}$ in the working line operating points is calculated with a constant total pressure ratio. This is motivated by the experience from a former optimization, where blockage by endwall separation in the stator hub region was generated, reducing the effective exit area. Thus the compressor was throttled, resulting in a relocation of the operating point toward higher efficiency - rewarded by the fitness. The shown correction of the efficiency has proven to effectively prevent such tendencies.

\section{Constraints}

The other requirements are treated with a region of interest (notation: ROI), a constraint in terms of a tolerance interval, which affects the Pareto-rank calculation. All deviations of specified parameters from its ROI-limits are summed up, the resulting value is minimized in a single objective optimization until all ROI are fulfilled. The ROI-intervals in the metamodel optimization are set more tightly than in
ROI1 (Exit Swirl Angle):
ROI2 (Mass Flow in OP2):

$$
\begin{aligned}
& \alpha_{\text {abs,StageExit,MassAveraged }} \leq 1^{\circ} \\
& \dot{m}_{\mathrm{OP} 2 \text {,target }} \pm 0.5 \%
\end{aligned}
$$
ROI3 (Mass Flow in OP3):
$\dot{m}_{\mathrm{OP} 3 \text {,target }} \pm 0.5 \%$

ROI4 (Von-Mises stress) :

with $\alpha_{a b s, \text { StageExit,MassAveraged }}=$

$\sum_{k=0}^{\mathrm{kmax}-1} 0.5 \cdot\left(\left|\alpha_{a b s, \text { StageExit }}(k)\right|+\left|\alpha_{a b s, \text { StageExit }}(k+1)\right|\right) \cdot\left(\dot{m}_{r e l}(k+1)-\dot{m}_{r e l}(k)\right)$ the CFD-evaluation branch. In the metamodel optimization the constraints directly affect optimization alignment. In the evaluation process the ROIs help the engineer identify members with unacceptable constraint violations.

ROI were set for the exit swirl angle by its mass averaged absolute value (avoiding compensatory effects), for the mass flow rates in the working line operating points OP2 and OP3, and for the maximum von-Mises stress found in the FE-analysis of the rotor blade. The absolute Mach number at stator exit is controlled by the area of the exit panel, thus by the parameterization.

\section{Discussion of Results}

\section{Pareto Front}

Figure 9 shows the status of the optimization and the Pareto front after 1250 convergent members (red symbols). Computational effort including the metamodel acceleration was about two months on 130 state of the art CPUs. Objective 2, the mean stall margin, is plotted over the mean working line stage efficiency, the first objective. This kind of diagram is commonly used in multi objective optimizations to observe the optimization progress and to identify dominant members. Smaller fitness values indicate

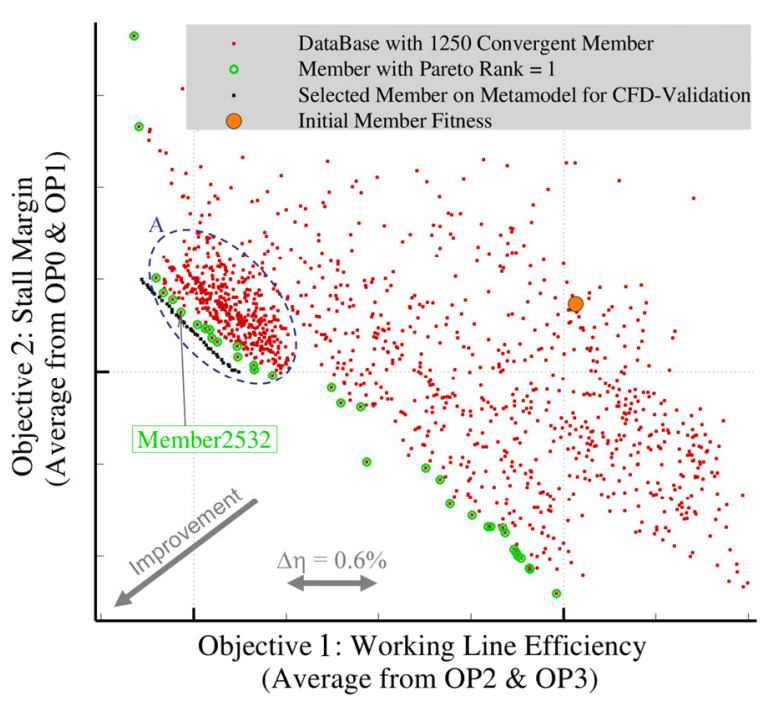

Figure 9. Pareto Front. Fitness Values for all Member in the Database and Metamodel Predictions. 
improvement; therefore superior members (with Pareto Rank 1, marked with green circles) are located toward the lower left corner. The fitness of the initial member, the starting point for this optimization, is denoted by the orange point. Black symbols represent predictions, generated by the Kriging algorithm at the end of the metamodel optimization. These members are to be evaluated by structural and CFD simulations.

In a first phase of the optimization a wider optimization interval for objective 2 was used, resulting in a broad distribution of the fitness values. Then the interval was reduced to maximize the progress in the region of target stability margin. A stripe pattern appears in that corridor (marked with "A" in figure 9), which represents members of one metamodel iteration and indicates the high prediction quality of the metamodel.

As final result of the optimization Member2532 was selected. It has about the same stall margin than the initial member (ignoring its mass flow deficit) but a $2.5 \%$ increased working line efficiency - a significant improvement. In the following the geometry and some aerodynamical aspects of this

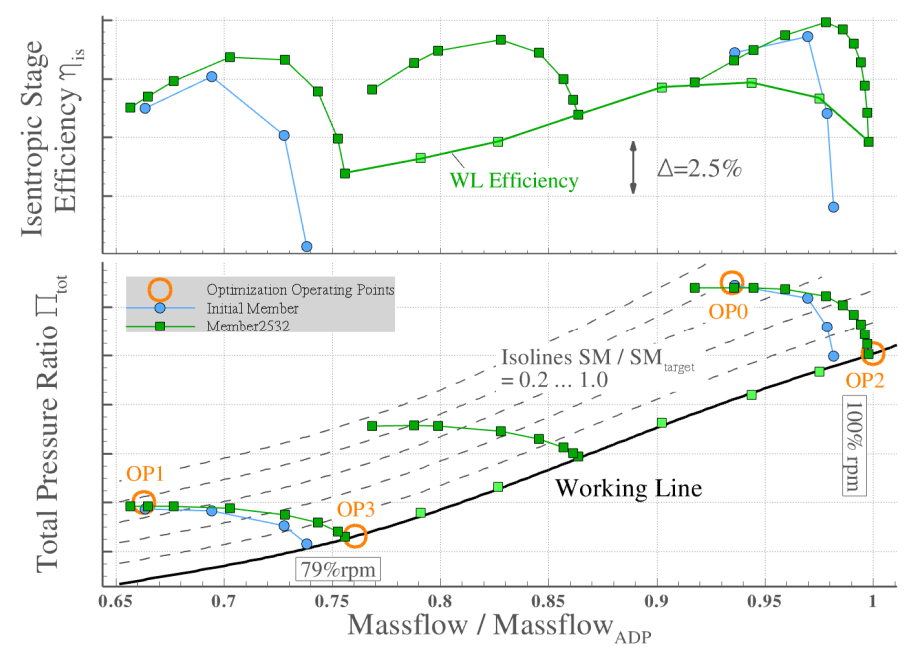

Figure 10. Performance Map. Member2532 in comparison to the Initial Member. member are presented.

\section{Performance Map}

Figure 10 shows the compressor performance map of Member2532 in comparison to the initial performance map. In addition to the optimization operating points, speedlines and the working line have better resolved with more simulated points. Member2532 mass flow rates in the working line operating points are now on target and the stall margin was raised by a broader working range with a similar near stall total pressure ratio. The intermediate speed line with $88 \%$ rpm indicates a constant stability margin for the compressor working range and supports the optimization concept with four operating points. Working line efficiency has increased significantly by $2.5 \%$ with about the same improvement at $100 \%$ and $79 \% \mathrm{rpm}$. These results prove that the described optimization strategy and setup successfully dealt with the complex interacting optimization goals.

\section{Geometries and Aerodynamics}

Hub und casing contour (figure 11) has not changed significantly due to former optimizations with a similar setup in the duct part. The first parameter-fitness correlations that the metamodels identify are for duct design parameters, due to their global impact on flow conditions. A note on the "bump" ahead of the rotor leading edge in the casing contour (marked with " $\mathrm{A}$ " in figure 11): removing this feature only slightly affected the mass flow rate due to the casing spline characteristics. This corresponds to the finding that the duct control points close to the rotor leading edge go to their upper limits resulting in the maximum casing radius. Furthermore, duct contouring has developed in the casing stator region ("C"), an adaptation to the new stator blade numbers.

The rotor leading edge ("B"), where only shifts of the control points in downstream direction were allowed, has

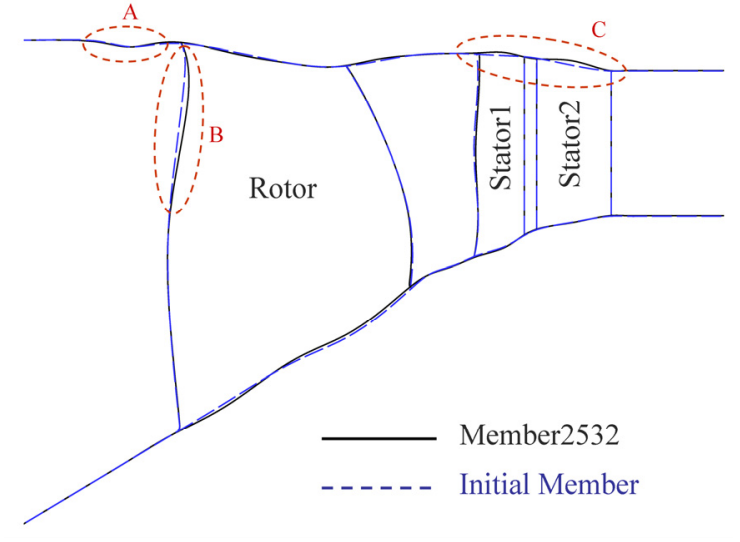

Figure 11. Duct Geometry.

Member2532 versus Initial Member

changed for a more pronounced forward sweep keeping the same tip chord length. Interestingly, the benefit of the different leading edge shape overcompensated the penalty of a shorter chord.

Figure 12 illustrates the 3D blading geometries. Due to a small hub-to-tip ratio (table 1) the rotor blade is highly twisted, has a low aspect ratio, and a moderate forward sweep. The shape of the rotor trailing edge of Member 2532 is more complex compared to the initial member. This indicates a change in the radial load distribution, found to be 
crucial for the performance improvements in the working line operating points (see radial distribution plot of stage isentropic efficiency in figure 13). Rotor incidence in the lower part has significantly changed to negative ("A" in the section Mach number distribution in figure 13), reducing the rotor total pressure ratio and thereby the shock strength and losses in the stator rows under working line conditions (left view in figure 13 with the isentropic surface Mach numbers on the blades). Stator

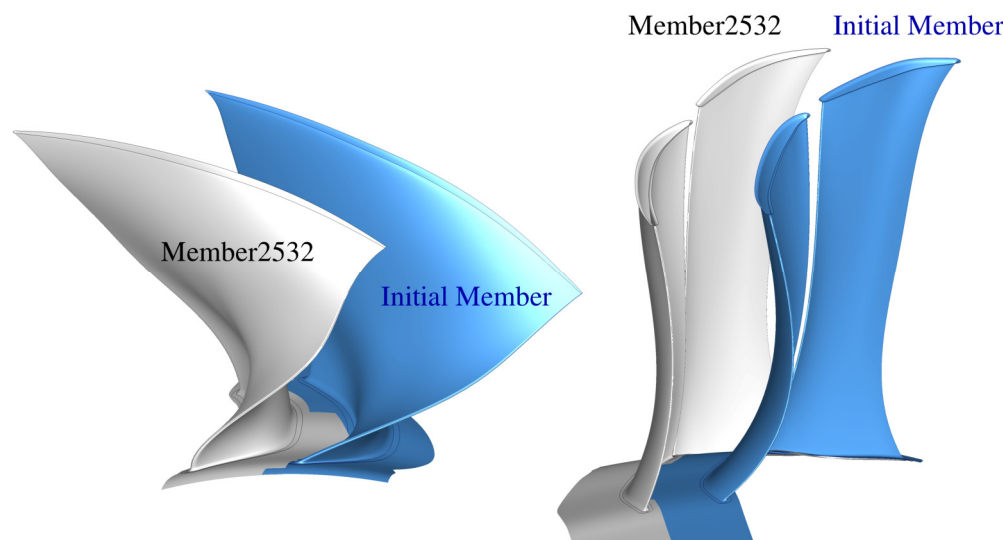

Figure 12. Blade Geometries. Member2532 versus Initial Member section profiles have adapted to inflow angles and shock positions, keeping the pre-shock Mach numbers low in the near stall operating points with high boundary layer loading.

Initially, the stronger bow in statorII resulted in a transport of low momentum fluid from the hub surface along the span, triggered by the statorI passage shock (working line OP) or high diffusion and the LE shock close to stall conditions. At some blade height that fluid joined with the wake of statorI, resulting in a greater separation zone (marked with "B" in the lower right Mach contour plots in figure 13).

Member2532 resolved this issue by reducing the bow and using the statorI wake "body" to accelerate the flow in between the wake and the blade surfaces. This mechanism together with a slightly more separated relative stator positioning (blade-to-blade Mach number distributions in figure 13) resulted in an about centered, isolated statorI wake at statorII trailing edge with proper flow conditions close to the blade surfaces.

Above $30 \%$ span the tandem stator of Member 2532 works perfectly with a balanced loading between the stator rows and the statorI wake "dissolves" in the statorII passage, energized by the statorII suction side potential field. Close to the hub, the aerodynamic demands to be fulfilled by a stator system are extreme. The improvements achieved here by this optimization run represent almost the maximum possible under the given constraints: constant blade numbers, the axial gap with no stator overlapping, and no exchange of axial length between the two stator rows. Future work will tend to make these features accessible by optimization.

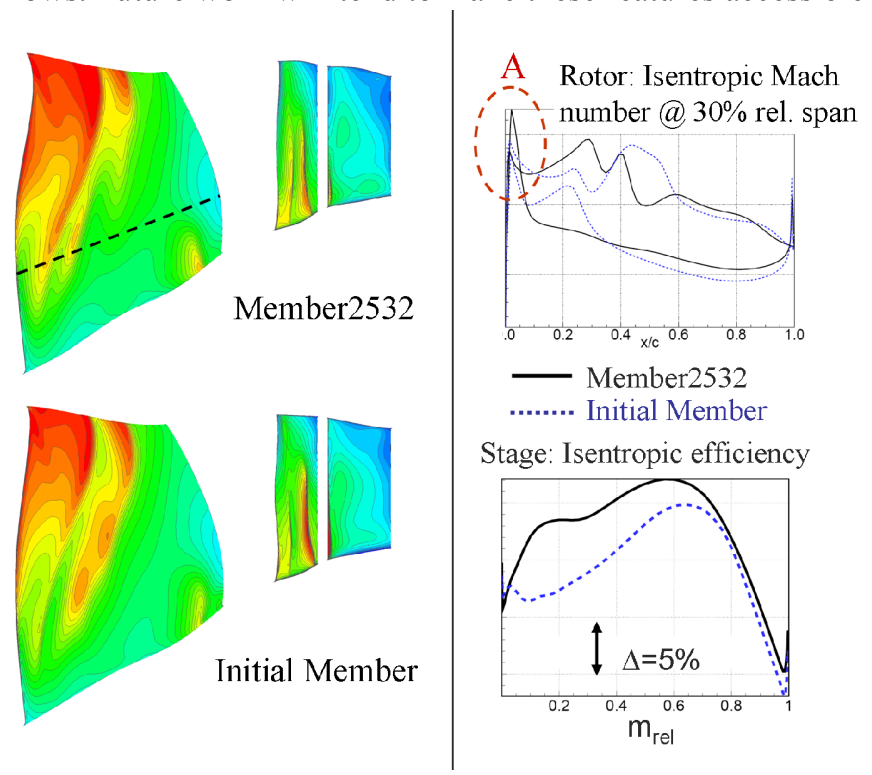

Figure 13. Mach Number Distributions.

Left: $\quad$ Mach contour plot of blade suction sides;

Center Left: Upper: Rotor section Mach number at $\mathrm{h}_{\text {rel }}=30 \%$; Lower: Radial distribution of stage isentropic efficiency;

Center Right: Stator blade-to-blade Mach contour at $\mathrm{h}_{\mathrm{rel}}=10 \%$; Right: $\quad$ Mach number in $\mathrm{x}=$ const. plane at statorII TE;

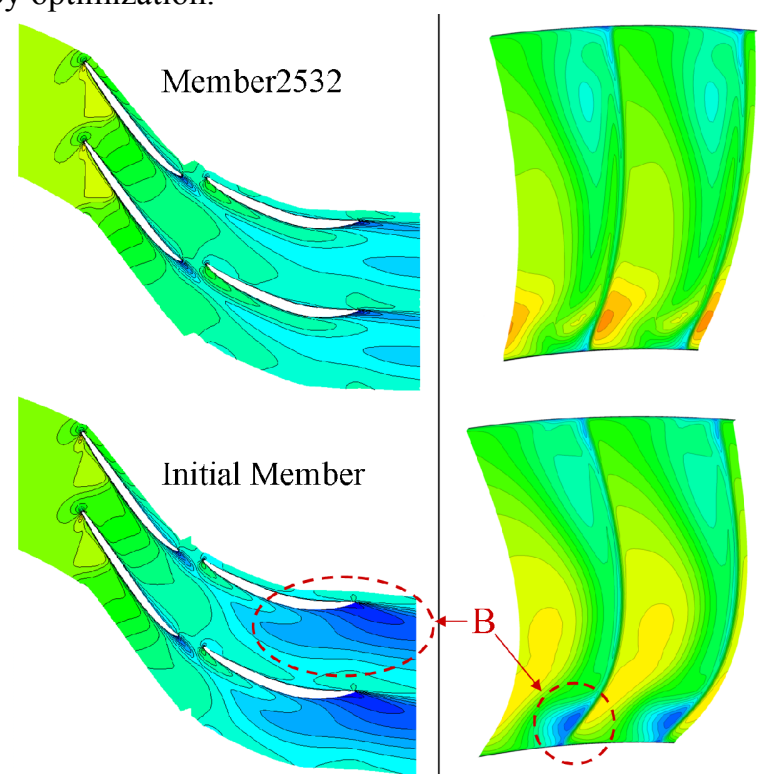

Operating point: Working line, $100 \%$ rpm Operating point: Working line, $100 \% \mathrm{rpm}$ Operating point: Working line, $100 \% \mathrm{rpm}$ Operating point: Near stall, $100 \% \mathrm{rpm}$ Operating point: Near stall, $100 \% \mathrm{rpm}$ 


\section{Conclusion}

A highly loaded, transonic axial compressor stage has been optimized with DLR's optimization process AutoOpti. Four aerodynamic operating points and a finite element analysis of the rotor blade were considered, enabling the optimization of both working line performance and stall margin for two rotational speeds under a mechanical feasibility constraint. Metamodel acceleration techniques allowed maximum design possibilities with the extremely high number of 231 free design parameters. Results show a significant improvement of the stage efficiency, extended stability margin while achieving the targeted mass flow rates and exit swirl angle. Including the finite element analysis of the rotor blade resulted in improved mechanical attributes.

\section{Acknowledgments}

The support of this work by the German Federal Ministry of Defence is gratefully acknowledged.

The authors also would like to thank the members of the numerics group for their invaluable help with all numerical related questions and Dr. Rainer Schnell for assisting with the implementation of the mass flow controller in TRACE.

\section{References}

${ }^{1}$ Holland, J. H., “Adaptation in natural and artificial systems”, Ann Arbor: The University of Michigan Press, 1975.

${ }^{2}$ Rechenberg, I., „Evolutionsstrategie - Optimierung technischer Systeme nach Prinzipien der biologischen Evolution“, Stuttgart: Frommann-Holzboog, 1973.

${ }^{3}$ Schwefel, H.-P., "Numerical optimization of computer models", Chichester: Wiley \& Sons, 1981.

${ }^{4}$ Fogel, D. B., "An Introduction to Simulated Evolutionary Optimization", IEEE Trans. on Neural Networks: Special Issue on Evolutionary Computation, Vol. 5, No. 1, pp. 3-14, 1994.

${ }^{5}$ Voß, C., Aulich, M., Kaplan, B., and Nicke, E., "Automated Multi-objective Optimization in Axial Compressor Blade Design”. ASME Paper GT2006-90420.

${ }^{6}$ Rai, M., "Multiple-Objective Optimization with Differential Evolution and Neural Networks", NASA Ames Research Centre, VKI Lecture-Series 2004.

${ }^{7}$ Ahmed, R., and Lawerenz, M., "On the AeroMechanical Design of Multistage Axial Compressors using Parallel Optimization Algorithms". 16’th Symposium on airbreathing engines, Number ISABE 2003-17.

${ }^{8}$ Keane, A.J., "Statistical Improvement Criteria for Use in Multiobjective Design Optimization", Automated Design: IMechE Seminar Proceedings 2006.

${ }^{9}$ Bellman, R.E. 1957. Dynamic Programming. Princeton University Press, Princeton, NJ.

${ }^{10}$ Dorfner, C., Nicke, E., Voß, C., "Axis-Asymmetric Profiled Endwall Design Using Multi-objective Optimization Linked with 3D-RANS-Flow-Simulations". ASME Paper GT2007-27268.

${ }^{11}$ Nürnberger, D., "Implizite Zeitintegration für die Simulation von Turbomaschinenströmungen". Dissertation. Fakultät für Maschinenbau der Ruhr-Universität Bochum. Auch ISRN DLR-FB-2004-27.

${ }^{12}$ Kügeler, E., Weber, A. and Lisiewicz, S., "Combination of a transition model with a twoequation turbulence model and comparison with experimental results"', Proc. 4th Eu. Conf. TurbMach, ATI-CST-076/01, Florence, Italy.

${ }^{13}$ Koulovic, D., „Modellierung des Grenzschichtumschlags bei Turbomaschinenströmungen unter Berücksichtigung mehrerer Umschlagsarten“, Dissertation. Fakultät für Maschinenbau der Ruhr-Universität Bochum. Auch ISRN DLR-FB-2007-20.

${ }^{14}$ Drela, M., Youngren, H.,"A User's guide to Mises”, MIT Computational Aerospace Sciences Laboratory, 1998

${ }^{15}$ Voß C., Becker K., "Multi-objective optimization in axial compressor design using a linked CFD-solver", ASME Paper GT2008-51131.

${ }^{16}$ Giannakoglou, K. C., and Karakasis, M. K., 2006. "Hierarchical and Distributed Metamodel-Assisted Evolutionary Algorithms". VKI Lecture Series 2006-03: Introduction to Optimization and Multidisciplianry Design.

${ }^{17}$ Chung, H.-S., and Alonso, J. J., "Multiobjective Optimization Using Approximation Model-Based Genetic Algorithms". 10th AIAA/ISSMO Symposium on Multidisciplinary Analysis and Optimization, AIAA 20044325. 